13,01

\title{
Фазовое равновесие и поверхностные характеристики в бинарной системе, содержащей наноразмерные частицы
}

\author{
(C) М.А. Шебзухова, А.А. Шебзухов \\ Кабардино-Балкарский государственный университет им. Х.М. Бербекова \\ Нальчик, Россия \\ E-mail: sh-madina@mail.ru
}

(Поступила в Редакцию 29 марта 2017 г.

В окончательной редакции 11 мая 2017 г.)

\begin{abstract}
В рамках классического метода с разделяющимися поверхностями дано согласованное описание фазового равновесия и поверхностных явлений в бинарных системах, содержащих монодисперсные наночастицы сферической формы произвольного (в том числе нанометрового) размера. С использованием полученных соотношений проведены расчеты состава сосуществующих фаз, межфазного слоя между ними, а также межфазное натяжение на границе наноразмерных частиц и матрицы при разных температурах в системе Ti-Мо. Результаты расчетов согласуются с имеющимися экспериментальными данными.
\end{abstract}

DOI: $10.21883 /$ FTT.2018.02.45398.100

\section{1. Введение}

В теории фазового равновесия в гетерогенных системах одно из центральных мест занимает вопрос о влиянии кривизны поверхности на характеристики сосуществующих фаз и межфазного слоя между ними [1-3]. Результаты исследований в данном направлении представляют значительный интерес для решения многих прикладных задач в области нанотехнологии и наноматериалов, а также целенаправленной модификации свойств макроскопических материалов путем создания в них дисперсных частиц $[4,5]$.

Многие направления исследований в данной области освещены в работах [6-8], а также в цитируемых в этих работах публикациях. При этом, наряду с известными подходами в рамках теории нуклеации с резкими (Беккер и Деринг, 1935 г.) и протяженными (Кан и Хиллард) границами раздела, значительный интерес, с нашей точки зрения, представляют фундаментальные работы по термодинамике фазовых равновесий в бинарных и многокомпонентных системах, включая термодинамику дисперсных систем при строгом учете поверхностных явлений [1-3]. Отметим особо факт использования в этих исследованиях представлений о межфазном слое конечной толщины и реальных величин, имеющих понятный физический смысл. Строгие термодинамические соотношения, в виде системы дифференциальных уравнений и неравенств, полученные в этих работах, позволили сформулировать ряд термодинамических закономерностей поверхностных и объемных процессов. Эти соотношения, как справедливо отмечает А.И. Русанов [1], образуют прочный фундамент для дальнейших теоретических исследований и анализа разнообразных моделей, используемых при описании фазовых равновесий и нахождении характеристик поверхностных слоев в гетерогенных системах.
При анализе результатов, полученных в рамках термодинамики фазовых равновесий и поверхностных явлений [1], возникают вопросы по обоснованности рассмотрения малых объектов в рамках термодинамического метода [5]. В связи с этим, отметим, следуя [1], что малым объектом здесь является одна из фаз (дисперсная фаза), а другая фаза (дисперсионная среда) имеет большие размеры и система в целом является макроскопической. Соотношения, полученные в рамках термодинамики фазовых равновесий и поверхностных явлений с использованием представлений о разделяющих поверхностях, как отмечено Гиббсом [9] и его последователями [1], справедливы даже в случае, когда малый объект не имеет собственной объемной части и может рассматриваться как состоящий целиком из поверхностного слоя (вплоть до обращения радиуса поверхности натяжения в нуль, когда внешняя фаза достигает границ устойчивости относительно непрерывных изменений, и работа образования новой фазы становится равной нулю [1]).

В работе [10] нами (в рамках теории фазовых равновесий с учетом поверхностных явлений) получена система уравнений в интегральном виде, позволяющая согласованным образом находить состав сосуществующих в равновесных условиях фаз, поверхностного слоя между ними и межфазное натяжение в бинарной системе с заданной степенью дисперсности одной из фаз в зависимости от температуры. Полученные новые соотношения были использованы для построения размернозависимой диаграммы состояния эвтектического типа с твердыми растворами с ОЦК- и ГПУ-структурами [10].

В настоящей работе приведены результаты аналогичных расчетов для бинарной системы, с непрерывными твердыми растворами, когда одна из фаз диспергирована в другой фазе (дисперсионная среда). Будем считать, что диспергированные частицы имеют одинаковый размер, который может находиться в том числе в нанодиапазоне, 
когда существенны внутренние и внешние степени свободы [3]. Кроме того, приведена система новых уравнений, позволяющая находить указанные выше величины для наночастиц, находящихся в равновесных условиях в матрице в изотермических условиях.

\section{2. Соотношения между основными параметрами состояния в бинарной наносистеме в изотермических условиях}

Основные исходные уравнения, приведенные в [10], получены в рамках классического варианта термодинамики поверхностных явлений в системах с искривленными границами при последовательном использовании концепции разделяющей поверхности и поверхностного натяжения [1]. В качестве разделяющей поверхности используется поверхность натяжения, которая, совместно с эквимолекулярной разделяющей поверхностью позволяет ввести в рассмотрение параметр Толмена. Эти уравнения могут быть рассмотрены при различных физических условиях.

Первое уравнение изотермы межфазного натяжения на границе наночастицы сферической формы (фаза $\alpha)$ и матрицы (фаза $\beta)$ в бинарной системе, полученные нами, имеет вид

$$
\sigma=\frac{R T r}{2 v_{01 \infty}^{(\alpha)}} m_{0}\left\{\ln \left[\frac{\kappa_{1}}{\kappa_{1 \infty}}\left(\frac{\kappa_{2 \infty}}{\kappa_{2}}\right)^{\rho_{\alpha \beta}}\right]+\Delta F\right\},
$$

где

$$
\begin{aligned}
& \Delta F= \sum_{n=\alpha, \beta}^{\prime} \frac{Q^{(n)}(r)}{R T}\left\{\left[\left(1-x_{1}^{(n)}\right)^{2}-\rho_{\alpha \beta}\left(x_{1}^{(n)}\right)^{2}\right]\right. \\
&\left.-\left[\left(1-x_{1 \infty}^{(n)}\right)^{2}-\rho_{\alpha \beta}\left(x_{1 \infty}^{(n)}\right)^{2}\right]\right\}, \\
& \kappa_{i}=x_{i}^{(\beta)} / x_{i}^{(\alpha)}, \quad \rho_{\alpha \beta}=\left(v_{0 i}^{(\beta)}-v_{0 i}^{(\alpha)}\right) /\left(v_{02}^{(\beta)}-v_{02}^{(\alpha)}\right), \\
& m_{0}=\left(1-v_{02}\right) /\left(1-v_{01} / v_{02}\right), \quad v_{0 i}=v_{0 i}^{(\beta)} / v_{0 i}^{(\alpha)},
\end{aligned}
$$

$x_{i}^{(\xi)}, v_{0 i}^{(\xi)}-$ мольная доля и молярный объем в чистом состоянии $i$-го компонента в фазе $\xi(\xi=\alpha, \beta), r-$ радиус поверхности натяжения, $Q(r)$ - параметр квазихимического взаимодействия (энергия смешения), $T-$ температура, $R$ - универсальная газовая постоянная. Величины, отмеченные нижним индексом „, $0^{6}$ и „, $\infty^{\text {“ }}$ указывают на принадлежность этих величин соответственно к чистому состоянию и макроскопической фазе. Штрих у суммы в (2) указывает на то, что перед вторым слагаемым (т.е. когда $n=\beta$ ) необходимо ставить знак минус. При получении (1) принималось во внимание слабая зависимость от радиуса поверхности натяжения величины $\Delta v=\rho_{\alpha \beta} v_{02}^{(\alpha)}-v_{01}^{(\alpha)}$, определяемой различием молярных объемов чистых компонентов.

Для систем с заданной степенью дисперсности в изотермических условиях можно получить еще одно уравнение изотермы межфазного натяжения, где фигурируют концентрации в сосуществующих фазах

$$
\sigma=\sigma_{02}+\frac{R \operatorname{Trn}_{0}}{2 v_{02}^{(\alpha)}(r)}\left\{\ln \left[\frac{\kappa_{1}}{\xi_{1}(r) \kappa_{2}^{\rho_{\alpha \beta}}}\right]+\Delta F_{0}\right\},
$$

где

$$
\begin{gathered}
\Delta F_{0}=\frac{Q^{(\alpha)}(r)}{R T}\left[1-\left(x_{2}^{(\alpha)}\right)^{2}+\rho_{\alpha \beta}\left(x_{1}^{(\alpha)}\right)^{2}\right] \\
-\frac{Q^{(\beta)}(r)}{R T}\left[1-\left(x_{2}^{(\beta)}\right)^{2}+\rho_{\alpha \beta}\left(x_{1}^{(\beta)}\right)^{2}\right], \\
n_{0} \approx n_{0 \infty}=\left(\gamma_{\nu \infty}^{(\alpha)}-\rho_{\alpha \beta \infty}\right), \\
\xi_{1}(r)=\lim \frac{x_{1}^{(\beta)}}{x_{1}^{(\alpha)}}, \quad \gamma_{\nu \infty}^{(\alpha)}=v_{01 \infty}^{(\alpha)} / \nu_{02 \infty}^{(\alpha)} .
\end{gathered}
$$

Значения межфазного натяжения наночастицы второго компонента в собственной матрице $\sigma_{02}$ в зависимости от радиуса поверхности натяжения в изотермических условиях могут быть рассчитаны с использованием формулы, полученной нами в работе [11]. Для размерной зависимости параметра квазихимического взаимодействия $Q^{(\xi)}(r)$ $(\xi=\alpha, \beta)$ и молярного объема $v_{02}^{(\alpha)}(r)$ можно использовать выражения, приведенные в [10].

Для величины $\xi_{1}(r)$ имеет место выражение

$$
\begin{aligned}
\xi_{1}(r) & =\exp \left\{\frac{\left(v_{01}^{(\beta)}-v_{01}^{(\alpha)}\right)}{R T}\left[p_{01}^{(\beta)}(r)-p_{02}^{(\beta)}(r)\right]\right. \\
& \left.+\frac{2 v_{01}^{(\alpha)}}{r R T}\left[\sigma_{02}(r)-\sigma_{01}(r)\right]+\frac{Q^{(\alpha)}(r)-Q^{(\beta)}(r)}{R T}\right\} .
\end{aligned}
$$

Значения для давления $p_{0 i}^{(\beta)}(r)$ в фазе $\beta$ в зависимости от радиуса поверхности натяжения, фигурирующего в (6), можно найти, пользуясь следующим соотношением

$$
p_{0 i}^{(\beta)}(r)=p_{0 i \infty}^{(\beta)}+\frac{2\left(v_{0 i \infty}^{(\alpha)} / v_{0 i \infty}^{(\beta)}\right)}{1-\left(v_{0 i \infty}^{(\alpha)} / v_{0 i \infty}^{(\beta)}\right)} \frac{\sigma_{0 i}(r)}{r},
$$

которое следует из обобщенного дифференциального уравнения Ван-дер-Ваальса для случая искривленной поверхности в однокомпонентной системе [1].

Соотношения (1) и (3) не имеют аналогов для случая плоской поверхности $(r=\infty)$ и для макроскопической системы они переходят в уравнение, которое связывает состав сосуществующих фаз $\alpha$ и $\beta$ в изотермических условиях в приближении регулярных растворов.

Для бинарной системы с заданной степенью дисперсности можно получить следующие уравнения, связывающие межфазное натяжение с составами сосуществующих фаз и межфазного слоя между ними в изотермических 
условиях

$$
\begin{aligned}
\sigma & =\sigma_{01}(r)+\frac{R T}{\omega_{1}(r)\left[1+\frac{2 \delta_{01}}{r}\left(1+\frac{\delta_{01}}{r}+\frac{1}{3} \frac{\delta_{01}^{2}}{r^{2}}\right)\right]} \\
& \times\left\{\ln \left[\frac{x_{1}^{(\sigma)}}{x_{1}^{(\alpha)}}\left(\frac{x_{1}^{(\alpha)}}{x_{1}^{(\beta)}}\right)^{\rho_{1}}\right]+\left(1-x_{1}^{(\sigma)}\right)^{2} \frac{Q^{(\sigma)}(r)}{R T}\right. \\
& \left.+\left(1-\rho_{1}\right)\left(1-x_{1}^{(\alpha)}\right)^{2} \frac{Q^{(\alpha)}(r)}{R T}-\rho_{1}\left(1-x_{1}^{(\beta)}\right)^{2} \frac{Q^{(\beta)}(r)}{R T}\right\},
\end{aligned}
$$

где $\rho_{1}=\left(1-v_{1}^{(\sigma)} / v_{1}^{(\alpha)}\right) /\left(1-v_{1}^{(\beta)} / v_{1}^{(\alpha)}\right), \delta_{01}$ - параметр Толмена для первого компонента. В рамках модели регулярных растворов, которая была использована при выводе (8), можно получить еще одно уравнение изотермы межфазного натяжения в виде

$$
\begin{aligned}
\sigma= & \sigma_{02}(r)+\frac{R T}{\omega_{2}(r)}\left[1+\frac{2 \delta_{02}}{r}\left(1+\frac{\delta_{02}}{r}+\frac{1}{3} \frac{\delta_{02}^{2}}{r^{2}}\right)\right]^{-1} \\
& \times\left\{\ln \left[\frac{x_{2}^{(\sigma)}}{x_{2}^{(\alpha)}}\left(\frac{x_{2}^{(\alpha)}}{x_{2}^{(\beta)}}\right)^{\rho_{1}}\right]+\left(x_{1}^{(\sigma)}\right)^{2} \frac{Q^{(\sigma)}(r)}{R T}\right. \\
& \left.+\left(1-\rho_{2}\right)\left(x_{1}^{(\alpha)}\right)^{2} \frac{Q^{(\alpha)}(r)}{R T}-\rho_{2}\left(x_{1}^{(\beta)}\right)^{2} \frac{Q^{(\beta)}(r)}{R T}\right\},
\end{aligned}
$$

где $\rho_{2}=\left(1-v_{2}^{(\sigma)} / v_{2}^{(\alpha)}\right) /\left(1-v_{2}^{(\beta)} / \nu_{2}^{(\alpha)}\right)$.

\section{3. Основные соотношения между параметрами состояния в бинарной системе с заданной степенью дисперсности в изобарических условиях $\left(p^{(\beta)}=\right.$ const $)$}

Для описания фазового равновесия двух конденсированных фаз бинарной системы в макроскопической системе часто используется условие постоянства давления $\left(p_{\infty}=\right.$ const $)$ [12]. Фазовые диаграммы состояния, которые широко используются на практике, являются изобарическими разрезами зависимости $T, P,\left\{x_{i}\right\}$. Следуя этому, зафиксируем давление во внешней фазе $\left(p^{(\beta)}=\mathrm{const}\right)$ и проведем рассмотрение для бинарной системы с заданной степенью дисперсности. Воспользуемся условиями равновесия фаз $\alpha$ и $\beta$

$$
\begin{gathered}
\frac{\partial g^{(\alpha)}}{\partial x_{i}^{(\alpha)}}=\frac{\partial g^{(\beta)}}{\partial x_{i}^{(\beta)}}, \\
g^{(\alpha)}-x_{i}^{(\alpha)} \frac{\partial g^{(\alpha)}}{\partial x_{i}^{(\alpha)}}=g^{(\beta)}-x_{i}^{(\beta)} \frac{\partial g^{(\beta)}}{\partial x_{i}^{(\beta)}},
\end{gathered}
$$

где $g^{(\xi)}$ - термодинамический потенциал фазы $\xi$ (на моль), $\xi=\alpha, \beta$. В результате будем иметь следующие соотношения

$$
\begin{gathered}
\frac{2 v_{0 i}^{(\sigma)}(r)}{r}\left[\sigma-\bar{\sigma}_{0 i}\left(r, \bar{T}_{0 i}\right)\right]=\left[\bar{s}_{0 i}^{(\beta)}\left(r, \bar{T}_{0 i}\right)-\bar{s}_{0 i}^{(\alpha)}\left(r, \bar{T}_{0 i}\right)\right] \\
\times\left[\bar{T}_{0 i}(r)-T\right]+R T \ln \frac{x_{i}^{(\beta)}}{x_{i}^{(\alpha)}} \\
+\left(1-x_{i}^{(\beta)}\right)^{2} Q^{(\beta)}(r)-\left(1-x_{i}^{(\alpha)}\right)^{2} Q^{(\alpha)}(r), \\
\frac{\left[\sigma-\sigma_{0 i}(r, T)\right] \bar{\omega}_{0 i}\left(r, T_{0 i}\right)}{R T}=\ln \frac{x_{i}^{(\sigma)}}{x_{i}^{(\alpha)}}-\tilde{\rho}_{0 i}\left(r, \bar{T}_{0 i}\right) \ln \frac{x_{i}^{(\beta)}}{x_{i}^{(\alpha)}} \\
+\left(1-x_{i}^{(\sigma)}\right)^{2} \frac{Q^{(\sigma)}(r)}{R T}-\left[1-\tilde{\rho}_{0 i}\left(r, \bar{T}_{0 i}\right)\right]\left(1-x_{i}^{(\alpha)}\right)^{2} \\
\quad \times \frac{Q^{(\alpha)}(r)}{R T}-\tilde{\rho}_{0 i}\left(r, \bar{T}_{0 i}\right)\left(1-x_{i}^{(\beta)}\right)^{2} \frac{Q^{(\beta)}(r)}{R T},
\end{gathered}
$$

где

$$
\begin{array}{r}
\tilde{\rho}_{0 i}\left(r, \bar{T}_{0 i}\right)=\bar{\rho}_{0 i}\left(r, \bar{T}_{0 i}\right)+\frac{\bar{\omega}_{0 i}\left(r, \bar{T}_{0 i}\right) \bar{\delta}_{0 i}}{\bar{v}_{0 i}^{(\alpha)}\left(r, \bar{T}_{0 i}\right)}\left(1+\frac{\bar{\delta}_{0 i}}{r}+\frac{1}{3} \frac{\bar{\delta}_{0 i}^{2}}{r^{2}}\right), \\
\sigma_{0 i}(r, T)=\bar{\sigma}_{0 i}\left(r, \bar{T}_{0 i}\right)+\left[T-\bar{T}_{0 i}(r)\right]\left(\frac{d \sigma_{0 i}}{d T}\right)_{p^{(\beta)}},
\end{array}
$$

$\bar{\delta}_{0 i}$ - параметр Толмена для границы фаз $\alpha$ и $\beta$ для $i$-го компонента в изобарических условиях $\left(p^{(\beta)}=\right.$ const $)$. Величины, отмеченные сверху чертой $\left(\bar{\sigma}_{0 i}, \bar{s}_{0 i}, \bar{T}_{0 i}, \bar{v}_{0 i}, \bar{\omega}_{0 i}, \bar{\delta}_{0 i}\right)$ относятся к температуре равновесия $\bar{T}_{0 i}(r)$ фаз $\alpha \quad$ и $\beta$ и все они зависят от радиуса поверхности натяжения. Для параметра Толмена обычно принимается его предельное значение при $r=\infty$ (для плоской поверхности) [1,13]. Для рассматриваемого случая, когда $p^{(\beta)}=$ const, нами показано, что $\bar{\delta}_{0 i}=\delta_{0 i}+\Delta \delta_{0 i}$, где $\delta_{0 i}=r_{e}-r$, $\Delta \delta_{0 i} \approx \frac{\bar{v}_{0 i \infty}^{(\alpha)}}{\left(\bar{s}_{0 i \infty}^{(\beta)}-\bar{s}_{0 i \infty}^{(\alpha)}\right)} \frac{d \sigma_{0 i \infty}}{d T}, r_{e}-$ радиус эквимолекулярной разделяющей поверхности.

Соотношения (10) и (11) можно записать для каждого компонента $(i=1,2)$ и таким образом получить четыре уравнения, в которых содержатся четыре неизвестных $x_{i}^{(\alpha)}, x_{i}^{(\beta)}, x_{i}^{(\sigma)}$ и $\sigma$. Нами проведены расчеты этих величин для бинарной системы Тi-Mo, компоненты которой имеют неограниченную растворимость в твердом (ОЦКструктура) и жидком состояниях [14].

\section{4. Входные данные, результаты расчетов и их краткое обсуждение}

В табл. 1 приведены все входные данные, использованные нами с указанием источников или расчетной формулы. На рисунке и в табл. 2 и 3 приведены результаты наших расчетов. Из них следует, что в макроскопическом случае $(r=\infty)$ результаты расчетов достаточно 
Таблица 1. Входные данные для расчета линий фазовых равновесий и межфазных характеристик в системе Ті-Мо в макроскопическом случае $(r=\infty)$ и с учетом наноразмерных эффектов $(r \neq \infty)$

\begin{tabular}{|c|c|c|}
\hline \multirow{2}{*}{ № } & \multicolumn{2}{|c|}{ Компоненты } \\
\hline & Тi (индекс 1) & Мо (индекс 2) \\
\hline 1 & $\bar{T}_{01 \infty}=1940 \mathrm{~K}[15]$ & $\bar{T}_{02 \infty}=2900 \mathrm{~K}[15]$ \\
\hline 2 & $\bar{S}_{01 \infty}^{(l)}-\bar{S}_{01 \infty}^{(\beta)}=8.3682 \cdot 10^{7} \frac{\mathrm{erg}}{\mathrm{mol} \cdot \mathrm{K}}[15]$ & $\bar{S}_{02 \infty}^{(l)}-\bar{S}_{02 \infty}^{(\beta)}=8.3682 \cdot 10^{7} \frac{\mathrm{erg}}{\mathrm{mol} \cdot \mathrm{K}}[15]$ \\
\hline 3 & $\bar{\sigma}_{01 \infty}=269 \mathrm{~mJ} / \mathrm{m}^{2}[15,16]$ & $\bar{\sigma}_{02 \infty}=464 \mathrm{~mJ} / \mathrm{m}^{2}[16]$ \\
\hline 4 & $\bar{v}_{01 \infty}^{(\beta)}=10.72 \mathrm{~cm}^{3} / \mathrm{mol}[15]$ & $\bar{v}_{02 \infty}^{(\beta)}=9.40 \mathrm{~cm}^{3} / \mathrm{mol}[16]$ \\
\hline 5 & $\bar{v}_{01 \infty}^{(l)}=10.9950 \mathrm{~cm}^{3} / \mathrm{mol}[15,17]$ & $\bar{v}_{02 \infty}^{(l)}=9.80 \mathrm{~cm}^{3} / \mathrm{mol}[15,17]$ \\
\hline 6 & $\begin{array}{c}\bar{\omega}_{01 \infty}=20.7035 \cdot 10^{7} \mathrm{~cm}^{2} \\
\text { (расчет в }[10])\end{array}$ & $\begin{array}{c}\bar{\omega}_{02 \infty}=19.0674 \cdot 10^{7} \mathrm{~cm}^{2} \\
(\text { расчет в }[10])\end{array}$ \\
\hline 7 & $\begin{array}{c}\left(\frac{d \sigma_{01 \infty}}{d T}\right)_{P^{(\beta)}}=0.0305 \frac{\mathrm{mJ}}{\mathrm{m}^{2} \mathrm{~K}} \\
\left(\text { рассчитано следуя }[16] \text { при } T=\bar{T}_{01 \infty}\right)\end{array}$ & $\begin{array}{c}\left(\frac{d \sigma_{02 \infty}}{d T}\right)_{P(\beta)}=0.0457 \frac{\mathrm{mJ}}{\mathrm{m}^{2} \mathrm{~K}} \\
\left(\text { рассчитано следуя }[16] \text { при } T=\bar{T}_{02 \infty}\right)\end{array}$ \\
\hline 8 & $\alpha_{01 \infty}=\frac{126}{3} \cdot 10^{-6} \mathrm{~K}^{-1}[18]$ & $\alpha_{02 \infty}=\frac{50}{3} \cdot 10^{-6} \mathrm{~K}^{-1}[18]$ \\
\hline 9 & $\begin{array}{c}\delta_{01}=0.0567 \cdot 10^{-7} \mathrm{~cm} \\
(\text { рассчитано следуя }[19])\end{array}$ & $\begin{array}{c}\delta_{02}=0.0544 \cdot 10^{-7} \mathrm{~cm} \\
(\text { рассчитано следуя [19]) }\end{array}$ \\
\hline 10 & $\begin{array}{c}\Delta \delta_{01}=0.0387 \cdot 10^{-7} \mathrm{~cm} \\
\left(\text { по формуле } \Delta \delta_{01}=\frac{\bar{v}_{01 \infty}^{(\beta)}}{{\overline{S_{01 \infty}^{(l)}}}^{(1)}-\bar{S}_{01 \infty}^{(\beta)}}\left(\frac{d \sigma_{01 \infty}}{d T}\right)_{P(\beta)}\right)\end{array}$ & $\begin{array}{c}\Delta \delta_{02}=0.0513 \cdot 10^{-7} \mathrm{~cm} \\
\left(\text { по формуле } \Delta \delta_{01}=\frac{\bar{v}_{01 \infty}^{(\beta)}}{\bar{S}_{01 \infty}^{(1)}-\bar{S}_{01 \infty}^{(\beta)}}\left(\frac{d \sigma_{01 \infty}}{d T}\right)_{P(\beta)}\right)\end{array}$ \\
\hline 11 & $\bar{\delta}_{01}=\delta_{01}+\Delta \delta_{01}=0.0954 \cdot 10^{-7} \mathrm{~cm}$ & $\bar{\delta}_{02}=\delta_{02}+\Delta \delta_{02}=0.1057 \cdot 10^{-7} \mathrm{~cm}$ \\
\hline $\begin{array}{l}12 \\
13\end{array}$ & $\begin{array}{r}r_{0}^{(\beta)}=0.2550 \cdot 10^{-} \\
Q_{\infty}^{(\beta)}=5192.46 \cdot 10^{7} \mathrm{erg} / \mathrm{mol}\end{array}$ & $\begin{array}{l}=0.2579 \cdot 10^{-7} \mathrm{~cm} \\
=6527.20 \cdot 10^{7} \mathrm{erg} / \mathrm{mol}[15]\end{array}$ \\
\hline
\end{tabular}

хорошо согласуются с экспериментальными данными для бинарной системы Тi-Мо [14]. В приближении идеальных растворов концентрация в поверхностном

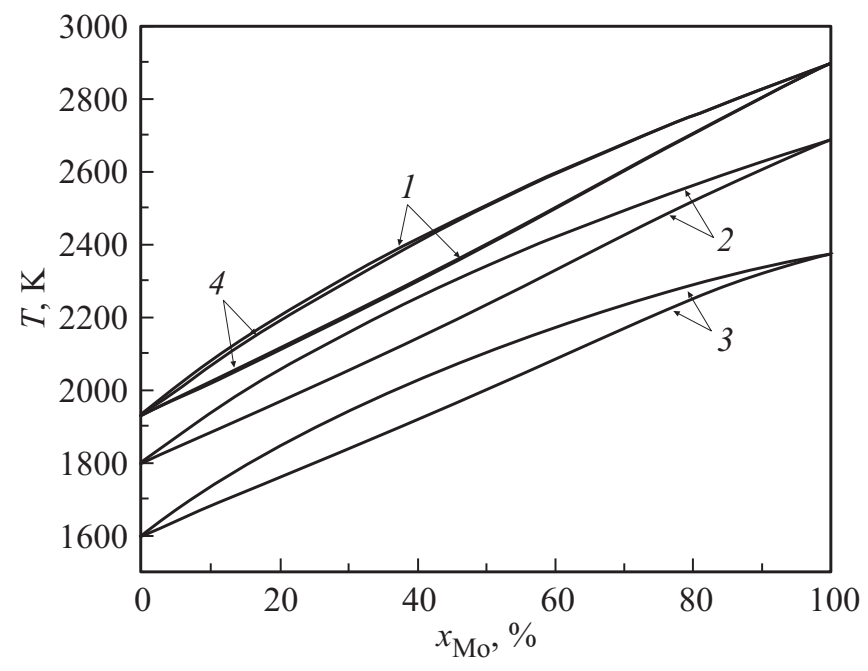

Диаграмма состояния бинарной системы Ті-Мо в макроскопическом случае $(1-r=\infty)$ и при наличии наноразмерных эффектов $(2-r=5 \mathrm{~nm}, 3-r=2 \mathrm{~nm}$ ) (расчет по формулам (10) и (11) с учетом межчастичных взаимодействий), (4- эксперимент, $r=\infty$, [14]). слое $x_{2 \infty}^{(\sigma)}$ между фазами с солидусными и ликвидусными концентрациями $x_{2 \infty}^{(\beta)}, x_{2 \infty}^{(l)}$ при температуре равновесия является промежуточной $\left(x_{2 \infty}^{(l)}<x_{2 \infty}^{(\sigma)}<x_{2 \infty}^{(\beta)}\right)$. Межфазное натяжение на границе указанных растворов с повышением температуры растет на всем интервале изменения температуры. Учет межчастичных взаимодействий (параметры взаимодействия в фазах имеют положительные значения и невелики по абсолютной величине) приводят к понижению содержания молибдена во всех фазах и межфазного натяжения при всех температурах.

При уменьшении радиуса наночастиц (до $15,10,5$ и $2 \mathrm{~nm}$ ) уменьшается температура равновесия (плавления) чистых компонентов, концентрация молибдена в сосуществующих фазах и в межфазном слое, а также межфазное натяжение на границе твердое тело-жидкость с солидусными и ликвидусными концентрациями.

При условиях $T=$ const и $p^{(l)}=$ const, как следует из табл. 1 , с уменьшением радиуса кривизны (радиуса поверхности натяжения) имеет место обогащение обеих фаз компонентом, содержание которого в дисперсной фазе больше по сравнению с матрицей (т.е. при $x_{\mathrm{Mo}}^{(\beta)}>x_{\text {Мо }}^{(l)}$ имеем $\left.\left(\frac{d x_{\mathrm{Mo}}^{(\beta)}}{d r}\right)_{T, P^{(l)}}<0,\left(\frac{d x_{\mathrm{Mo}}^{(l)}}{d r}\right)_{T, P^{(l)}}<0\right)$. Таким образом, наши расчеты подтверждают правило о таком влиянии искривления поверхности на состав сосуществующих фаз, сформулированное на основе ана- 
Таблица 2. Зависимость температуры и состава фаз, сосуществующих в равновесных условиях, в системе Ті-Мо в макроскопическом случае $(r=\infty)$ и при наличии наноразмерных эффектов $(r \neq \infty)$ (расчет по формулам $(10)$ и $(11)$ с учетом межчастичных взаимодействий)

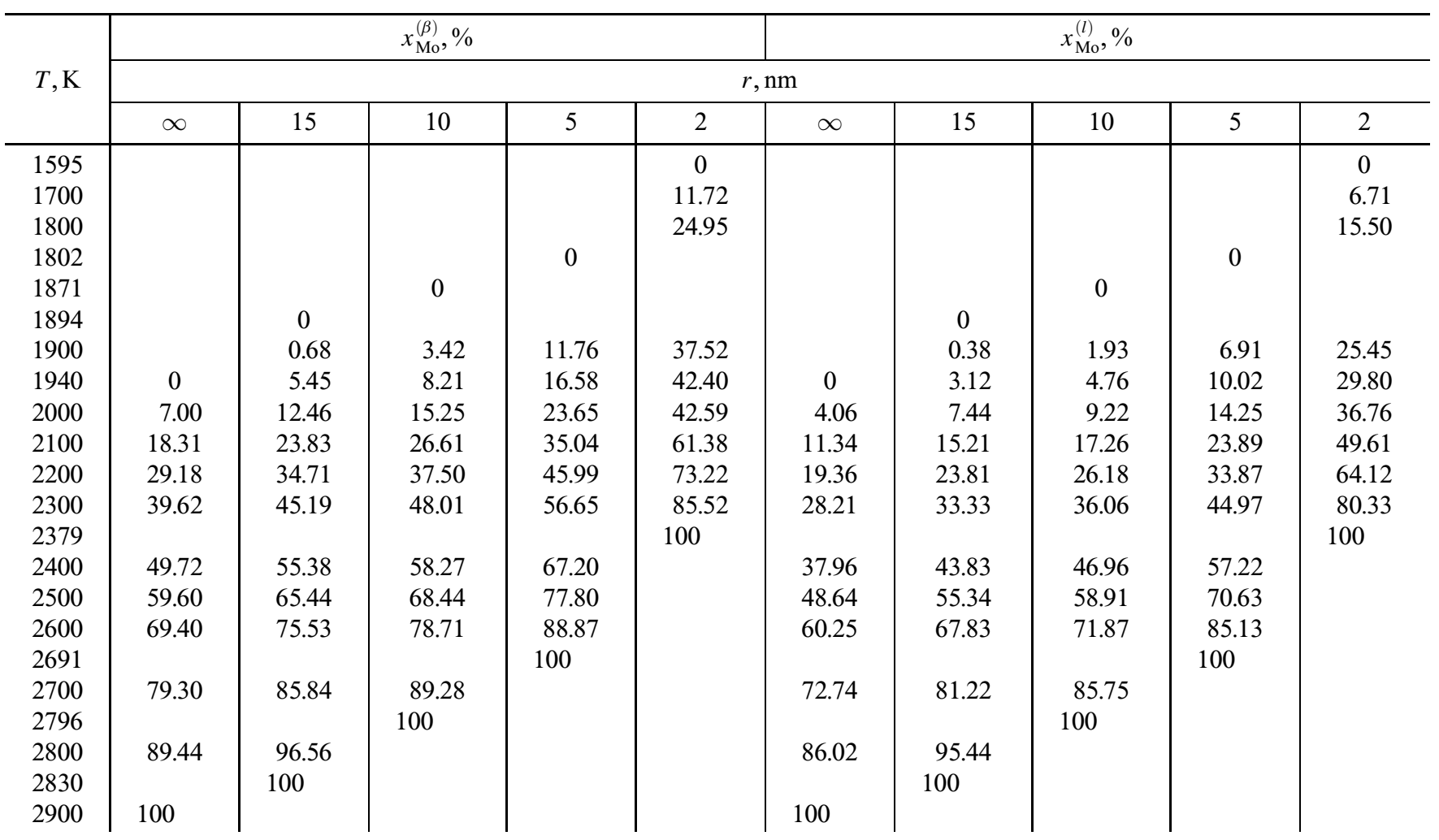

Таблица 3. Состав межфазного слоя и межфазное натяжение на границе твердое тело (ОЦК-структура)-жидкость в равновесных условиях в зависимости от температуры в системе Ті-Мо в макроскопическом случае $(r=\infty)$ и при наличии наноразмерных эффектов $(r \neq \infty)$ (расчет по формулам (10) и (11) с учетом межчастичных взаимодействий)

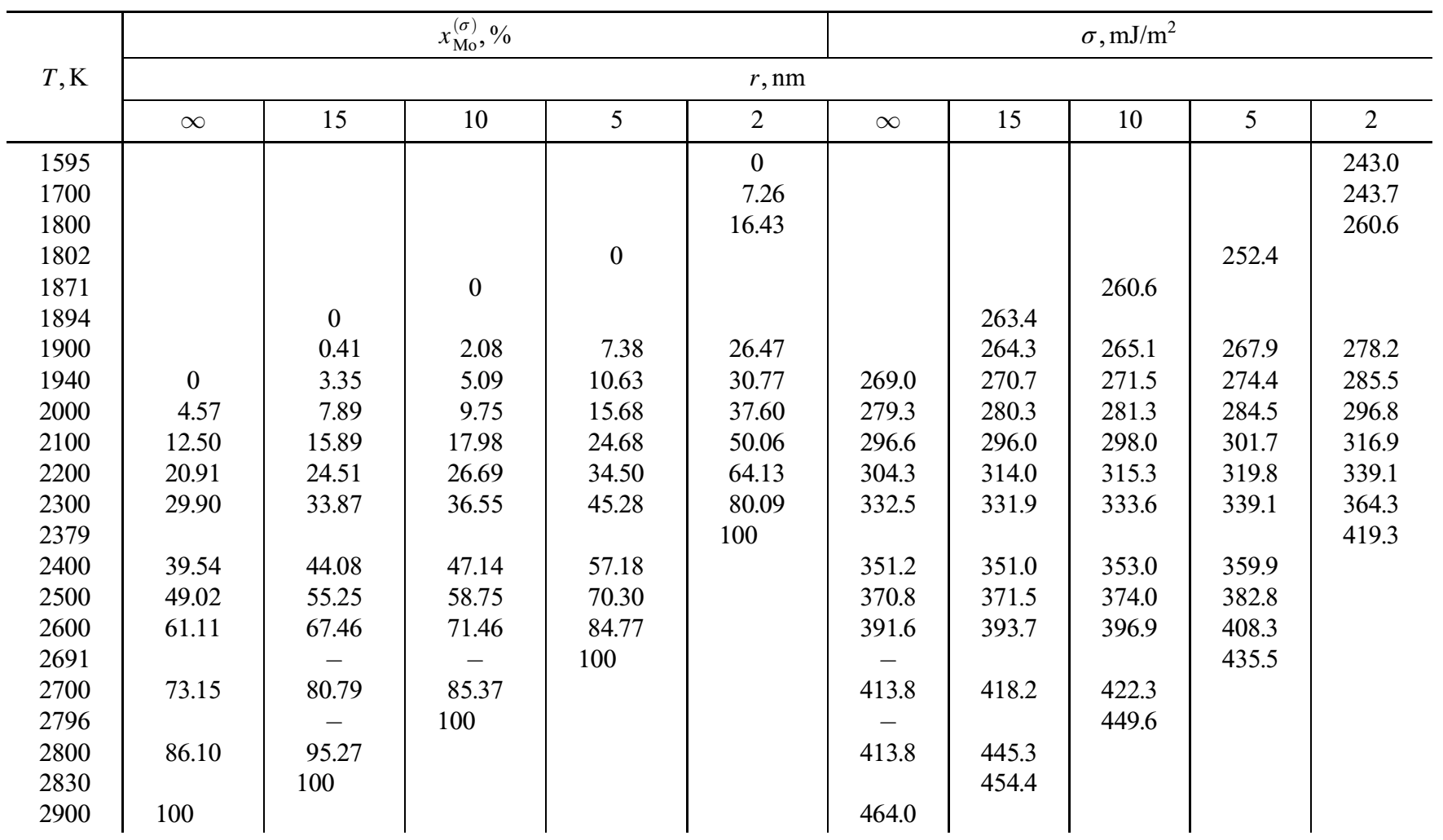


лиза фундаментальных уравнений термодинамики поверхностных явлений в дифференциальной форме [1].

Отметим наличие ряда данных (см., например, [6-8]), относящихся к твердым растворам, содержащим наноразмерные выделения вторых фаз, для которых в изотермических условиях характерно антибатное изменение состава матрицы и дисперсной фазы с изменением размера нановыделений. В подобных случаях, следовательно, производные $\left(\frac{d x_{i}^{(m)}}{d r}\right)_{T}$ и $\left(\frac{d x_{i}^{(n)}}{d r}\right)_{T}$ имеют разные знаки $\left(x_{i}^{(m)}, x_{i}^{(n)}-\right.$ концентрация $i$-го компонента в матрице и нановыделении соответственно). Такие данные, относящиеся к твердофазному состоянию, несомненно представляют интерес. При этом, по нашему мнению, целесообразно проводить проверку экспериментальных данных о равновесии сосуществующих фаз (матрицы и нанообъекта) и адекватности моделей, используемых в теоретических расчетах. Подобная практика (термодинамическая проверка) широко распространена при обработке опытных данных о фазовом равновесии в макроскопических системах [20].

\section{5. Заключение}

С единых позиций проведено рассмотрение размерной зависимости основных параметров равновесия (объемных и поверхностных) в бинарных системах, состоящих из матрицы макроскопического размера и монодисперсных частиц сферической формы.

Приведены выражения для согласованного расчета состава сосуществующих фаз и переходного слоя между ними, а также межфазного натяжения в зависимости от радиуса поверхности натяжения в изотермических и изобарических условиях. При устремлении размера дисперсных частиц к бесконечности из них в качестве частных случаев следуют известные выражения для состава сосуществующих объемных фаз, плоского поверхностного слоя и межфазного натяжения в бинарной системе.

Полученные соотношения использованы для расчета объемных и поверхностных характеристик фазового равновесия в системе Тi-Мо, состоящей из твердой фазы с ОЦК-структурой и жидкой матрицы с учетом наноразмерных эффектов и в макроскопическом случае при разных температурах в равновесных условиях. При проведении вычислений использованы наиболее надежные входные данные из литературы и способы учета размерных зависимостей индивидуальных характеристик компонентов и параметров межчастичного взаимодействия в фазах.

Установлено снижение положений линий ликвидуса и солидуса по температурной оси на диаграмме состояния при возрастании степени диспергирования твердого раствора в жидкой матрице и изменение состава сосуществующих в равновесных условиях фаз. Изменяются также состав поверхностного слоя и межфазное натяжение с изменением радиуса поверхности натяжения монодисперсных частиц. Размерные эффекты наиболее ярко выражены при переходе к более малым размерам дисперсных частиц.

В изотермо-изобарических условиях характер изменения состава сосуществующих фаз и межфазного слоя между ними с уменьшением размера наночастицы сферической формы (радиуса поверхности натяжения), полученные по результатам наших расчетов, подтверждают выводы, сформулированные на основе анализа фундаментальных уравнений термодинамики поверхностных явлений и дисперсных систем.

\section{Список литературы}

[1] А.И. Русанов. Фазовые равновесия и поверхностные явления. Химия, Л. (1967). 388 с.

[2] А.И. Русанов. Термодинамические основы механохимии. Наука, СПб. (2006). 221 с.

[3] А.И. Русанов. Лекции по термодинамике поверхностей. Лань, СПб. (2013). 240 с.

[4] Нанонаука и нанотехнологии. Энциклопедия систем жизнеобеспечения. Юнеско, EOLSS, Магистр-Пресс, М. (2010). 992 c.

[5] В.К. Григорович. Жаропрочность и диаграмма состояния. Металлургия, М. (1969). 324 с.

[6] П.Е. Львов, В.В. Светухин, А.В. Обухов. ФТТ 53, 2, 394 (2011).

[7] П.Е. Львов, В.В. Светухин. ФТТ 55, 11, 2256 (2013).

[8] П.Е. Львов, В.В. Светухин. ФТТ 56, 9, 1825 (2014).

[9] Дж.В. Гиббс. Термодинамические работы. Гостехиздат, М. (1950). 492 c.

[10] М.А. Шебзухова, А.А. Шебзухов. ФТТ 59, 7, 1368 (2017).

[11] 3.А. Шебзухов, М.А. Шебзухова, А.А. Шебзухов. Поверхность. Поверхность. Рентгеновские, синхротронные и нейтронные исследования. 11, 102 (2009).

[12] А.А.Смирнов. Молекулярно-кинетическая теория металлов. Наука, М. (1966). 488 с.

[13] 3.А. Шебзухов, М.А. Шебзухова, А.А Шебзухов. Известия Кабардино-Балкарского государственного университета им. Х.М. Бербекова. Нальчик, 1, 17 (2010).

[14] А. Хансен, К. Андерко. Структура двойных сплавов. Том 2. Металлургиздат, М. (1962). 1488 с.

[15] Л.Кауфман, Х.Бернстейн. Расчет диаграмм состояния с помощью ЭВМ. Мир, М. (1972). 326 с.

[16] Q. Jiang, H.M. Li. Surf. Sci. Rep. 63, 427 (2008).

[17] С.В. Станкус. Изменение плотности элементов при плавлении. Методы и экспериментальные данные. Препринт № 247-91. Новосибирск. (1991). 78 с.

[18] М.Н. Магомедов. Изучение межатомного взаимодействия, образования вакансий и самодиффузии в кристаллах. Физматлит, М. (2010), 544 с.

[19] W. Vogelsberger, G. Marx. Z. Phys. Chem. 257, 3, 580 (1976).

[20] В.Б. Коган. Гетерогенные равновесия. Химия, Л. (1968), $432 \mathrm{c}$. 